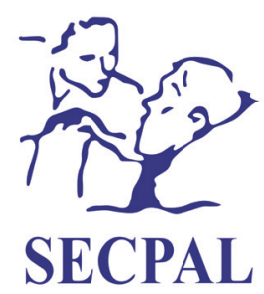

Medicina Paliativa

www.medicinapaliativa.es

ORIGINAL BREVE

\title{
Obstinación diagnóstica en pacientes oncológicos hospitalizados en situación de últimos días
}

\author{
María Ángeles González-Benítez ${ }^{* 1}$, María Luisa Amaya González ${ }^{1}$, Monserrat \\ Godoy Guerrero', Aurora Ruz Zafra ${ }^{1}$, Cristina Segura Schultz ${ }^{2}$ y Alberto Ruiz-Cantero ${ }^{1}$
}

\author{
${ }^{1}$ Unidad de Gestión Clínica, Medicina Interna, Hospital de la Serranía, Ronda, Málaga, España. ²Equipo de Soporte \\ de Cuidados Paliativos, Hospital de la Serranía, Ronda, Málaga, España
}

Recibido el 24 de julio de 2018

Aceptado el 24 de septiembre de 2018

PALABRAS CLAVE

Cuidados paliativos, obstinación

diagnóstica, final de

la vida.

\begin{abstract}
Resumen
Introducción: La limitación del esfuerzo terapéutico debería incluir la limitación de la realización de pruebas diagnósticas que no mejoran los síntomas del paciente. El objetivo de este estudio fue analizar el uso de pruebas diagnósticas en pacientes oncológicos hospitalizados en sus últimos días de vida.

Métodos: Se realizó un estudio descriptivo retrospectivo incluyendo todos los pacientes oncológicos estadio IV que fallecieron en el Servicio de Medicina Interna durante un periodo de dos años. Las variables analizadas fueron edad, sexo, número de pruebas de laboratorio (contabilizadas como una sola prueba por cada extracción), número de pruebas radiológicas (radiología simple, ecografía, tomografía computerizada [TC], resonancia magnética [RM]) y si se realizó extracción de analítica de sangre en las últimas 48 horas de vida.

Resultados: Fueron incluidos 51 pacientes (15 mujeres, 29,4\%), con una edad media de 71,94 años (desviación estándar 11,06). Se realizaron pruebas de laboratorio a 45 pacientes $(88,4 \%)$, con una mediana de 3 pruebas por paciente (rango intercuartílico 1,5). En 41 casos $(80,4 \%)$ se realizaron estudios radiológicos, con una mediana de 1 (rango intercuartílico 1,3) estudio por paciente. Las pruebas radiológicas realizadas fueron: radiografía simple (37 pacientes, $72,5 \%$ ), ecografía (17 pacientes, 33,3\%), TC (12 pacientes, 23,6\%), RM (1 paciente, $2 \%$ ). A 23 pacientes $(45,1 \%)$ se les realizó una extracción de sangre en las últimas 48 horas de vida. Conclusiones: Se realiza un número elevado de pruebas diagnósticas en los últimos días de vida de pacientes oncológicos en situación terminal. Aunque las pruebas realizadas no se consideran invasivas, producen molestias innecesarias a los pacientes que no contribuyen al control de síntomas.
\end{abstract}

*Autor para correspondencia:

María Ángeles González Benítez

Facultativo Especialista de Área, Servicio de Medicina Interna, Hospital de la Serranía. Carretera Ronda-San Pedro, km 2, 29400, Ronda, Málaga, España

Correo electrónico: mglezbenitez@yahoo.es, mariaa.gonzalez.benitez.sspa@juntadeandalucia.es 
KEYWORDS

Palliative care, diagnostic obstinacy, end of life.

\begin{abstract}
Introduction: Any limitation of therapeutic effort should include a limitation of diagnostic tests not contributing to patient comfort. The aim of the present study was to assess the use of diagnostic tests among hospitalized cancer patients during their last days of life.

Methods: A retrospective observational study was performed on all stage-IV cancer patients who died during hospitalization in the Internal Medicine Unit over a period of two years. Analyzed variables included: age, gender, number of laboratory tests, and number of imaging tests (plain $X$-rays, ultrasound, computed tomography [CT], magnetic resonance imaging [MRI]), and whether a blood test had been performed within the last 48 hours of life.

Results: A total of 51 patients were included (women, 15; $29.4 \%$ ) with an average age of 71.94 years (standard deviation, 11.06). Of these, 45 patients $(88.4 \%)$ underwent laboratory tests with a median of 3 tests per patient (interquartile range, 1.5), 41 patients ( $80.4 \%$ ) underwent imaging tests with a median of 1 test per patient (interquartile range, 1.3). The frequencies of imaging tests were: plain X-rays, 37 patients (72.5\%); ultrasounds, 17 patients (33.3\%); CT, 12 patients (23.6\%); MRI, 1 patient (2\%). Twenty-three patients $(45.1 \%)$ underwent at least one blood test within their last 48 hours of life.

Conclusions: A large number of laboratory and imaging tests are performed during the last days of terminally ill, hospitalized cancer patients. While these tests are not considered to be invasive, they cause unnecessary discomfort to patients, and do not contribute to symptom relief.
\end{abstract}

González-Benitez MA, Amaya González ML, Godoy Guerrero M, Ruz Zafra A, Segura Schultz C, Ruiz-Cantero A. Obstinación diagnóstica en pacientes oncológicos hospitalizados en situación de últimos días. Med Paliat. 2019;26(3):223-226.

\section{Introducción}

La obstinación terapéutica se define como la situación en la que a una persona, que se encuentra en situación terminal o de agonía y afecta de una enfermedad grave e irreversible, se le inician o mantienen medidas de soporte vital u otras intervenciones carentes de utilidad clínica, que únicamente prolongan su vida biológica, sin posibilidades reales de mejora o recuperación, siendo, en consecuencia, susceptibles de limitación". Dentro de "otras intervenciones carentes de utilidad clínica" no solo estarían incluidos tratamientos, sino también pruebas diagnósticas que no aportan beneficios al paciente y que, sin embargo, conllevan molestias, riesgos e indicación de tratamientos innecesarios. La realización de dichas pruebas podría considerarse obstinación diagnóstica.

La población atendida en nuestro centro (hospital comarcal) se encuentra muy dispersa geográficamente y el acceso a los cuidados paliativos en domicilio difiere de forma importante de unos municipios a otros. Esto motiva que un número importante de pacientes con enfermedad terminal pasen sus últimos días ingresados en la Unidad de Medicina Interna. Al estar hospitalizados, el acceso a las pruebas diagnósticas es fácil y rápido. Sin embargo, dado que lo más probable es que dichas pruebas no estén indicadas, su realización podría considerarse obstinación diagnóstica y sería, por lo tanto, susceptible de limitación. En estudios previos sobre órdenes de limitación del esfuerzo terapéutico en pacientes hospitalizados en servicios de medicina interna, solo en el $5 \%$ de los casos se incluyó una orden de "no realización de pruebas diagnósticas", sin especificarse el tipo de pruebas incluidas en dicha orden ${ }^{2,3}$.
El objetivo de este trabajo es conocer el uso de pruebas diagnósticas en pacientes oncológicos hospitalizados en situación de últimos días en el Servicio de Medicina Interna de un hospital comarcal.

\section{Material y métodos}

Se realizó un estudio descriptivo incluyendo a todos los pacientes ingresados por enfermedad neoplásica terminal que fallecieron durante su ingreso en la Unidad de Medicina Interna durante los años 2013 y 2014. Fueron excluidos del estudio aquellos pacientes en los que el diagnóstico de la enfermedad oncológica se realizó durante el mismo ingreso en el que fallecieron, por considerar que en estos casos la realización de pruebas estaba justificada por el proceso diagnóstico. La recogida de datos se realizó de forma retrospectiva.

Las variables analizadas fueron edad, sexo, días de estancia hospitalaria, número de pruebas de laboratorio (contabilizadas como una sola prueba por cada extracción), número de pruebas radiológicas (radiología simple, ecografía, tomografía computerizada [TC], resonancia magnética [RM]) y si se realizó extracción de analítica de sangre en las últimas 48 horas de vida. Se incluyeron todas las pruebas diagnósticas realizadas desde el momento del ingreso en planta de hospitalización. No fueron incluidas las realizadas en el Servicio de Urgencias.

Las variables numéricas fueron resumidas mediante medias y desviaciones estándar (DE), utilizándose mediana y rango intercuartílico (RI) en caso de distribuciones asimétricas. Las cualitativas se resumieron mediante frecuencias y porcentajes. 


\section{Resultados}

Desde el 1 de enero de 2013 hasta el 31 de diciembre de 2014 fallecieron 56 pacientes por enfermedad neoplásica terminal en nuestra unidad. Fueron excluidos del estudio 5 pacientes por tratarse de casos en los que la enfermedad neoplásica fue diagnosticada en el mismo ingreso durante el que se produjo el fallecimiento.

Fueron incluidos en el estudio 51 pacientes (15 mujeres, $29,4 \%$, y 36 hombres, 70,6\%), con una edad media de 71,94 años (DE 11,06). La mediana de estancia hospitalaria fue de seis días $(\mathrm{RI} 3,17,5)$. Se realizaron pruebas de laboratorio a 45 pacientes $(88,4 \%)$, con una mediana de tres pruebas por paciente (RI 1,5). A 23 pacientes $(45,1 \%)$ se les realizó una extracción de sangre en las últimas 48 horas de vida (Tabla I). En 41 casos $(80,4 \%)$ se realizaron estudios radiológicos, con una mediana de $1(\mathrm{RI} 1,3)$ estudio por paciente. Las pruebas radiológicas realizadas fueron, en orden de frecuencia: radiografía simple (37 pacientes, $72,5 \%$ ), ecografía (17 pacientes, 33,3\%), TC (12 pacientes, 23,6 \%), RM (1 paciente, $2 \%$ ) (Tabla II).

\section{Discusión}

El número de pruebas diagnósticas realizadas parece excesivo dado que se trata de pacientes oncológicos en sus últimos días de vida, en los que el objetivo del tratamiento es conseguir un buen control sintomático.

Existe escasa literatura sobre el uso de pruebas diagnósticas en los últimos días de vida. Nuestros hallazgos van en la misma línea que los obtenidos por West y cols. en un estudio en el que comparaban la administración de fármacos y la realización de pruebas diagnósticas y terapéuticas en los últimos tres días de vida a pacientes oncológicos ingresados en hospitales de agudos frente a centros de cuidados paliativos (hospice). La realización de gasometría arterial, radiografía simple, ecografía y TC fue significativamente mayor en los hospitales, donde los pacientes tenían tres veces más probabilidades de que se le realizara uno o más estudios diagnósticos ${ }^{4}$.

En el estudio de Belloni y cols. sobre la solicitud de exámenes radiológicos en pacientes con enfermedad oncológica terminal, al $86 \%$ de los pacientes estudiados se les sometió al menos a un estudio radiológico en los 90 días previos a su ingreso en un centro de cuidados paliativos (Hospice), con una media de 4 (DE 3,2) estudios por paciente. Tras su ingreso, solo el $25 \%$ de los pacientes fueron sometidos a pruebas radiológicas, con una drástica reducción del núme-

Tabla I. Pruebas de laboratorio

\begin{tabular}{lc}
\hline $\begin{array}{l}\text { N. }{ }^{\circ} \text { de pacientes con pruebas de } \\
\text { laboratorio }\end{array}$ & $45(88,4 \%)$ \\
\hline Total de pruebas de laboratorio realizadas & 279 \\
\hline $\begin{array}{l}\text { Mediana de pruebas por paciente } \\
\text { Percetil 25, percentil 75 }\end{array}$ & 3 \\
\hline $\begin{array}{l}\text { N. }{ }^{\circ} \text { de pacientes con extracción de sangre } \\
\text { en las últimas } 48 \text { horas de vida }\end{array}$ & $23(45,1 \%)$ \\
\hline
\end{tabular}

ro de pruebas por paciente (media de 0,8, DE 0,4) ${ }^{5}$. En el trabajo de Hu y cols. sobre el uso de pruebas de imagen de alto coste (TC, RM, tomografía por emisión de positrones o medicina nuclear) en pacientes ancianos con enfermedad oncológica estadio IV, al 31,2 \% de los pacientes estudiados se les realizó al menos una de estas pruebas en su último mes de vida6.

En el estudio de Ameneiros-Lago y cols. sobre los últimos días de vida de pacientes con fallecimiento previsible ingresados en Medicina Interna, en el 23,5 \% de los casos se realizan procedimientos diagnósticos o terapéuticos no encaminados al control de síntomas, sin especificar el tipo de procedimiento ${ }^{7}$.

Las principales limitaciones de nuestro estudio son el tamaño pequeño de la muestra y la falta de definición sobre la indicación de la realización de estudios diagnósticos. La recogida de datos de forma retrospectiva trataba de evitar que la realización del estudio influyera en la toma de decisiones respecto a la solicitud de pruebas. Sin embargo, esto impide valorar adecuadamente el motivo de la solicitud de cada prueba y la indicación de cada una de ellas de forma aislada. Por tanto, no podemos saber qué porcentaje de las pruebas realizadas estaban realmente justificadas. No existe una definición establecida de cuándo un estudio diagnóstico está indicado o no, aunque podemos considerar que no es necesario realizarlo si su resultado no va a cambiar el manejo del paciente o, en caso de modificarlo, no le va a aportar beneficios. Es poco probable que la realización de pruebas mejorara el control de síntomas en los pacientes estudiados. Cabe argumentar que la solicitud de pruebas estaba justificada al comienzo del ingreso si aún no estaba claro que se trataba de una situación de últimos días. Por dicho motivo se incluyó en el diseño del estudio el análisis del número de extracciones de sangre en las últimas 48 horas de vida del paciente, objetivándose que ocurre en casi la mitad de los casos analizados. En el estudio de West y cols., al 16,7 \% de los pacientes oncológicos ingresados en hospitales de agudos se les realizó gasometría arterial en los últimos tres días de vida ${ }^{4}$.

Aunque solo fueron incluidos pacientes oncológicos para que la muestra fuera lo más homogénea posible, en nuestra unidad atendemos con mayor frecuencia a pacientes no oncológicos. Sería interesante analizar el uso de pruebas diagnósticas en los pacientes que fallecen por enfermedad

Tabla II. Estudios radiológicos

\begin{tabular}{lc}
\hline N. ${ }^{\circ}$ de pacientes con estudios radiológicos & $41(80,4 \%)$ \\
\hline Total de estudios radiológicos realizados & 135 \\
\hline Mediana de estudios por paciente & 1 \\
Percentil 25, percentil 75 & 1,3 \\
\hline Radiografía simple, n. $^{\circ}$ de pacientes & $37(72 \%)$ \\
\hline Ecografía, n. ${ }^{\circ}$ de pacientes & $17(33,3 \%)$ \\
\hline TC, n. ${ }^{\circ}$ de pacientes & $12(23,6 \%)$ \\
\hline RM, n. ${ }^{\circ}$ de pacientes & $1(2 \%)$ \\
\hline
\end{tabular}


crónica avanzada, en los que se suele retrasar la identificación como paciente paliativo ${ }^{8}$ y puede ser difícil establecer si se trata de una agudización grave o de una situación de últimos días. Probablemente en estos casos se solicite un mayor número de estudios complementarios.

\section{Conclusiones}

Se realiza un número elevado de pruebas diagnósticas en los últimos días de vida de pacientes oncológicos en situación terminal. Aunque las pruebas realizadas no se consideran cruentas ni invasivas, producen molestias a los pacientes (dolor por la extracción de sangre, traslados para realización de estudios radiológicos, etc.) que no contribuyen al control de sus síntomas, principal objetivo del tratamiento en estos casos. Además, elevan innecesariamente el coste de la atención a estos pacientes. Sería importante reflexionar sobre la utilidad de las pruebas diagnósticas en estas circunstancias, ampliar los estudios sobre este tema y ser conscientes de que la limitación del esfuerzo terapéutico incluye limitar la realización de pruebas diagnósticas innecesarias, aunque no se consideren invasivas.

\section{Conflicto de intereses}

Los autores declaran que no existe ningún tipo de conflicto de intereses. El presente trabajo se ha realizado sin fuentes de financiación.

\section{Bibliografía}

1. Ley $2 / 2010$, de 8 de abril, de Derechos y Garantías de la Persona en el Proceso de la Muerte. Publicado en BOJA, n. ${ }^{\circ} 88$, de 7 de mayo de 2010.

2. García Caballero R, Herreros B, Real de Asúa D, Gámez S, Vega G, García Olmos L. Limitación del esfuerzo terapéutico en pacientes hospitalizados en servicios de medicina interna. Rev Clin Esp. 2018;218:1-6.

3. García Caballero R, Herreros B, Real de Asúa D, Alonso R, Barrera $M M$, Castilla V. Limitación del esfuerzo terapeútico en pacientes hospitalizados en el Servicio de Medicina Interna. Rev Calid Asist. 2016;31:70-5.

4. West E, Costantini M, Pasman H, Onwuteaka-Philipsen B; EURO IMPACT. A comparison of drugs and procedures of care in the Italian hospice and hospital settings: the final three days of life for cancer patients. BMC Health Serv Res. 2014;14:496.

5. Belloni E, Tentoni S, Cella A, Cassinelli D, Bertè R, Scagnelli P. Radiological exams on end-stage oncologic patients before hospice admission. Radiol Med. 2017;122:793-7.

6. Hu YY, Kwok AC, Jiang W, Taback N, Loggers ET, Ting GV, et al. High-cost imaging in elderly patients with stage IV cancer. J Natl Cancer Inst. 2012;104:1164-72.

7. Ameneiros-Lago E, Carballada-Rico C, Garrido-Sanjuán JA. Mortalidad esperable en Medicina Interna: ¿cómo son los últimos días de vida en los pacientes en los que la muerte es previsible? Med Paliat. 2017;24:63-71.

8. Priego-Valladares M, González de la Aleja P, García-Navarro MM, Tello-Valero A, Portilla-Sogorb J, Ramos-Rincón JM. Diferencias en pacientes oncológicos y no oncológicos ingresados en una unidad de cuidados paliativos de reciente creación en un hospital de tercer nivel en la Comunidad Valenciana (España). Med Paliat. 2018;25:130-5. 